\title{
A Class of Integral Operators that Fix Exponential Functions
}

\author{
Carlo Bardaro, Ilaria Mantellini®, Gumrah Uysal and \\ Basar Yilmaz
}

\begin{abstract}
In this paper we introduce a general class of integral operators that fix exponential functions, containing several recent modified operators of Gauss-Weierstrass, or Picard or moment type operators. Pointwise convergence theorems are studied, using a Korovkin-type theorem and a Voronovskaja-type formula is obtained.
\end{abstract}

Mathematics Subject Classification. Primary 42C15, Secondary 46E22, 94A20.

Keywords. Convolution integrals, Exponential moments, Weighted moduli of continuity, Voronovskaja formula.

\section{Introduction}

The classical Bohman-Korovkin theorem (see $[10,22,23])$ is one of the pivotal results of approximation theory and several convergence theorems known in literature employ this basic tool. It states that a sequence of positive linear operators $T_{n} f$ acting on the set of the continuous functions over a compact interval of the real line converges to the identity operator only if it converges on a finite number of test functions which form a so-called Chebyshev system. A complete treatment of the Korovkin theorem can be found in the monographies $[2,3]$.

In this respect, if a sequence of operators $T_{n} f$ is such that $T_{n} \varphi=\varphi$ for some continuous function $\varphi$, then to obtain the convergence appears very simple, if the functions $\varphi$ belong to a Chebyshev system. Thus, it is of interest to define sequences of operators that have this property. In literature the so-called King type operators, have this property, especially in case of discrete operators (see e.g. [21]). In this paper we define an entire class of

Carlo Bardaro and Ilaria Mantellini have been partially supported by the "Gruppo Nazionale per l'Analisi Matematica e Applicazioni (GNAMPA)" of the "Instituto di Alta Matematica (INDAM)" as well as by the project "Ricerca di Base 2019 of University of Perugia (title: Integrazione, Approssimazione Analisi non Lineare e loro Applicazioni)". 
positive linear integral operators which fix exponential functions. This kind of results were obtained by Agratini, Aral and Deniz [1], by Aral [6] and recently by Yilmaz, Uysal and Aral [26], by considering modifications of specific operators (Picard, Gauss-Weiertsrass and moment-type operators). All the operators considered in these papers are special case of our present theory. Our approach includes also all the integral operators having a compactly supported kernel. Thus it applies for example to spline-type operators [11,24]. Related results can be found in [16,18,19] and [17].

For our operators, we apply the Gadjiev version of Bohman-Korovkin theorem (see $[14,15])$ in case of unbounded domains, for functions belonging to certain weighted spaces of continuous functions. Setting for every $n \in \mathbb{N}$ and $a>0$,

$$
A_{a, n}:=\int_{-\infty}^{\infty} \exp (a t) K_{n}(t) \mathrm{d} t, \quad \lambda_{n}(x):=x-\frac{1}{a} \log A_{a, n},
$$

we define

$$
\left(T_{n} f\right)(x)=A_{a, n} \int_{-\infty}^{\infty} \exp (-a t) f\left(\lambda_{n}(x)+t\right) K_{n}(t) \mathrm{d} t
$$

where $\left\{K_{n}\right\}$ is a family of non-negative functions (kernel) belonging to a suitable function space. Setting for $a>0$, $\exp _{a}(x):=e^{a x}$ for $x \in \mathbb{R}$, we show that $\left(T_{n} \exp _{a}\right)(x)=\exp (a x)$ and $\left(T_{n} \exp _{2 a}\right)(x)=\exp (2 a x)$. Moreover we show that $T_{n} e_{j} \rightarrow e_{j}$ where $e_{j}(x)=x^{j}, j=0,1,2$, so obtaining two uniform convergence theorems in weighted spaces of continuous functions. Then, using certain moduli of continuity we obtain certain quantitative estimates of the convergence and finally a Voronovskaja-type asymptotic formula. These kinds of asymptotic formulae are very useful also for applications, especially for discrete operators, like e.g. sampling-type operators (see, e.g., [11-13]. For integral operators of Mellin type see $[7,8])$. The last section is devoted to several examples.

We recall here, that general approaches to convergence of integral operators was recently given in [9], and more recently in [20] and [25] in the frame of nonlinear operators.

\section{Basic Notations}

Let us denote by $\mathbb{R}$ the set of real numbers, and by $\mathbb{N}$ the set of the positive integers. By $L^{1}(\mathbb{R})$ we denote the space comprising all the Lebesgue integrable functions on $\mathbb{R}$ with respect to the Lebesgue measure, and by $L^{\infty}(\mathbb{R})$ the space comprising all the essentially bounded functions on $\mathbb{R}$. By $C(\mathbb{R})$ we denote the space of all the continuous functions defined on $\mathbb{R}$. Finally, for $r \in \mathbb{N}$, we will say that a function $f: \mathbb{R} \rightarrow \mathbb{R}$ is locally of class $C^{r}$ at a point $x \in \mathbb{R}$ if there is a neighbourhood $U$ of $x$ such that $f$ is $(r-1)$-fold continuously differentiable in $U$ and $f^{(r)}(x)$ exists. 


\section{The Class of Integral Operators}

For any $a>0$, we define the function $\exp _{a}(t):=e^{a t}$, for $t \in \mathbb{R}$.

We define now the function space

$$
L_{\exp _{a}}(\mathbb{R}):=\left\{g: \mathbb{R} \rightarrow \mathbb{R}: g(\cdot) e^{a|\cdot|} \in L^{1}(\mathbb{R})\right\} .
$$

Let now $\left\{K_{n}\right\}$ be a non negative kernel, that is, for every $n \in \mathbb{N}, K_{n}(t) \geq$ 0 , for every $t \in \mathbb{R}, K_{n} \in L^{1}(\mathbb{R})$, and

$$
\left\|K_{n}\right\|_{1}=\int_{-\infty}^{\infty} K_{n}(t) \mathrm{d} t=1 .
$$

In what follows we assume that $K_{n} \in L_{\exp _{a}}(\mathbb{R})$, for every sufficiently large $n \in \mathbb{N}$, namely for $n \geq n_{0}$, with $n_{0} \in \mathbb{N}$. We set, for $n \geq n_{0}$,

$$
A_{a, n}:=\int_{-\infty}^{\infty} \exp _{a}(t) K_{n}(t) \mathrm{d} t, \quad \lambda_{n}(x):=x-\frac{1}{a} \log A_{a, n} .
$$

Note that from the assumption $K_{n} \in L_{\exp _{a}}(\mathbb{R})$, we have $A_{a, n}<+\infty, A_{-a, n}<$ $+\infty$ and if moreover for every $n \in \mathbb{N}, K_{n}$ is an even function, that is $K_{n}(t)=K_{n}(-t)$, for every $t \in \mathbb{R}$, one has easily $A_{a, n}=A_{-a, n}$. We introduce now the sequence of integral operators defined by

$$
\begin{aligned}
\left(T_{n} f\right)(x) & :=A_{a, n} \int_{-\infty}^{\infty} \exp _{-a}(t) f\left(\lambda_{n}(x)+t\right) K_{n}(t) \mathrm{d} t \\
& =A_{a, n} \int_{-\infty}^{\infty} e^{-a t} f\left(\lambda_{n}(x)+t\right) K_{n}(t) \mathrm{d} t
\end{aligned}
$$

for every function $f$ belonging to the domain $D:=\bigcap_{n \geq n_{0}} \operatorname{dom} T_{n}$, where $\operatorname{dom} T_{n}$ denotes the set of all the Lebesgue measurable functions $f$ such that $\left(T_{n}|f|\right)(x)$ is convergent for almost all $x \in \mathbb{R}$.

Note that if $f \in L^{\infty}(\mathbb{R})$, then $f \in D$. Moreover, the functions $\exp _{a}$ and $\exp _{2 a}$ both belong to $D$. We have the following

Proposition 3.1. Under the established assumptions on the kernel $\left\{K_{n}\right\}$, we have

$$
\left(T_{n} \exp _{a}\right)(x)=\exp _{a}(x), \quad\left(T_{n} \exp _{2 a}\right)(x)=\exp _{2 a}(x) .
$$

Proof. We have

$$
\begin{aligned}
\left(T_{n} \exp _{a}\right)(x) & =A_{a, n} \int_{-\infty}^{\infty} e^{-a t} e^{a\left(\lambda_{n}(x)+t\right)} K_{n}(t) \mathrm{d} t=A_{a, n} e^{a \lambda_{n}(x)} \int_{-\infty}^{\infty} K_{n}(t) \mathrm{d} t \\
& =A_{a, n} e^{a \lambda_{n}(x)}=\exp _{a}(x) .
\end{aligned}
$$

Analogously, we have

$$
\begin{aligned}
\left(T_{n} \exp _{2 a}\right)(x) & =A_{a, n} \int_{-\infty}^{\infty} e^{-a t} e^{2 a\left(\lambda_{n}(x)+t\right)} K_{n}(t) \mathrm{d} t \\
& =A_{a, n} e^{2 a \lambda_{n}(x)} \int_{-\infty}^{\infty} e^{a t} K_{n}(t) \mathrm{d} t \\
& =A_{a, n} \exp _{2 a}(x) \frac{A_{a, n}}{\left(A_{a, n}\right)^{2}}=\exp _{2 a}(x),
\end{aligned}
$$

that is the assertion 
Remark 3.2. In order to establish a connection with classical integral operators of convolution type of the form

$$
\left(T_{n}^{0} f\right)(x):=\int_{-\infty}^{\infty} f(x+t) K_{n}(t) \mathrm{d} t,
$$

for continuous and bounded functions $f \in D$ we have

$$
\lim _{a \rightarrow 0^{+}}\left(T_{n} f\right)(x)=\left(T_{n}^{0} f\right)(x) .
$$

whenever

$$
\lim _{a \rightarrow 0^{+}} \frac{1}{a} \log A_{a, n}=0
$$

Indeed, under the above assumptions on the kernel $\left\{K_{n}\right\}$, it is easy to show that $\lim _{a \rightarrow 0^{+}} A_{a, n}=1$, and $\lim _{a \rightarrow 0^{+}} \lambda_{n}(x)=x$. Thus under the assumptions on the function $f$ the assertion follows by the Lebesgue theorem of dominated convergence.

\section{Pointwise and Uniform Convergence}

In this section we will study the pointwise convergence of $T_{n} f$ to $f$, where $f$ belongs to a suitable weighted space of continuous functions, as introduced in [14], using a Korovkin-type theorem, established in [14] (see also [15]). In order to do that, we introduce the following constants

$$
\begin{aligned}
& B_{-a, n}:=\int_{-\infty}^{\infty} t e^{-a t} K_{n}(t) \mathrm{d} t \\
& C_{-a, n}:=\int_{-\infty}^{\infty} t^{2} e^{-a t} K_{n}(t) \mathrm{d} t .
\end{aligned}
$$

We call these constants the exponential moments of orders 1 and 2 respectively. At the same way, we refer to $A_{-a, n}$ as the exponential moment of order 0 . We introduce the following subspace of $L_{\exp _{a}}(\mathbb{R})$, in which the above moments are well-defined

$$
L_{\exp _{a}}^{*}(\mathbb{R}):=\left\{g \in L_{\exp _{a}}(\mathbb{R}):(c+d|\cdot|)^{2} e^{a|\cdot|} g(\cdot) \in L^{1}(\mathbb{R})\right\},
$$

with $c, d$ positive constants. If $K_{n} \in L_{\exp _{a}}^{*}(\mathbb{R})$, then $B_{-a, n}, C_{-a, n}$ exist finite.

Let us introduce now the test functions $e_{0}(t)=1, e_{1}(t)=t, e_{2}(t)=$ $t^{2}, t \in \mathbb{R}$. Obviously, $e_{j} \in D$, for $j=0,1,2$.

We have the following

Proposition 4.1. Let $K_{n} \in L_{\exp _{a}}^{*}(\mathbb{R})$, for sufficiently large $n \in \mathbb{N}$. Then we have

$$
\begin{aligned}
& \left(T_{n} e_{0}\right)(x)=A_{a, n} A_{-a, n} \\
& \left(T_{n} e_{1}\right)(x)=A_{a, n} A_{-a, n} x-\frac{1}{a} A_{a, n} A_{-a, n} \log A_{a, n}+A_{a, n} B_{-a, n}
\end{aligned}
$$

and 


$$
\begin{aligned}
\left(T_{n} e_{2}\right)(x)= & A_{a, n} A_{-a, n} x^{2}+\left(2 A_{a, n} B_{-a, n}-2 A_{a, n} A_{-a, n} \frac{1}{a} \log A_{a, n}\right) x \\
& +A_{a, n} A_{-a, n} \frac{1}{a^{2}} \log ^{2} A_{a, n}+A_{a, n} C_{-a, n}-2 A_{a, n} B_{-a, n} \frac{1}{a} \log A_{a, n} .
\end{aligned}
$$

Proof. We have

$$
\left(T_{n} e_{0}\right)(x)=A_{a, n} \int_{-\infty}^{\infty} e^{-a t} K_{n}(t) \mathrm{d} t=A_{a, n} A_{-a, n} .
$$

Next,

$$
\begin{aligned}
\left(T_{n} e_{1}\right)(x) & =A_{a, n} \int_{-\infty}^{\infty} e^{-a t}\left(\lambda_{n}(x)+t\right) K_{n}(t) \mathrm{d} t \\
& =A_{a, n} \lambda_{n}(x) \int_{-\infty}^{\infty} e^{-a t} K_{n}(t) \mathrm{d} t+A_{a, n} \int_{-\infty}^{\infty} t e^{-a t} K_{n}(t) \mathrm{d} t \\
& =A_{a, n} A_{-a, n} x-A_{a, n} A_{-a, n} \frac{1}{a} \log A_{a, n}+A_{a, n} B_{-a, n} .
\end{aligned}
$$

Finally,

$$
\begin{aligned}
\left(T_{n} e_{2}\right)(x)= & A_{a, n} \int_{-\infty}^{\infty} e^{-a t}\left(\lambda_{n}(x)+t\right)^{2} K_{n}(t) \mathrm{d} t \\
= & A_{a, n}\left(\lambda_{n}(x)\right)^{2} A_{-a, n}+A_{a, n} C_{-a, n}+2 A_{a, n} B_{-a, n} \lambda_{n}(x) \\
= & A_{a, n} A_{-a, n} x^{2}+2 A_{a, n}\left(B_{-a, n}-\frac{1}{a} A_{-a, n} \log A_{a, n}\right) x \\
& +A_{a, n}\left(A_{-a, n} \frac{1}{a^{2}} \log ^{2} A_{a, n}+C_{-a, n}-\frac{2}{a} B_{-a, n} \log A_{a, n}\right) .
\end{aligned}
$$

The proof is completed

Corollary 4.2. Under the assumptions of Proposition 4.1, if moreover

$$
\lim _{n \rightarrow+\infty} A_{a, n}=\lim _{n \rightarrow+\infty} A_{-a, n}=1,
$$

and

$$
\lim _{n \rightarrow+\infty} B_{-a, n}=\lim _{n \rightarrow+\infty} C_{-a, n}=0
$$

then

$$
\lim _{n \rightarrow+\infty}\left(T_{n} e_{j}\right)(x)=e_{j}(x) \quad(j=0,1,2) .
$$

Proof. It is an immediate consequence of Proposition 4.1

Using Proposition 4.1 and Corollary 4.2, we now give a uniform convergence result for the operators $\left(T_{n} f\right)$ when $f$ belongs to suitable weighted spaces of continuous functions. The key tool is a Korovkin-type theorem proved in [14] (see also [15]).

Given a continuous, strictly increasing function $\varphi: \mathbb{R} \rightarrow \mathbb{R}$, we define the function $\rho(x):=1+\varphi^{2}(x)$, and assume that $\lim _{x \rightarrow \pm \infty} \rho(x)=+\infty$.

We will consider two particular cases: $\varphi_{1}(x)=x$, and $\varphi_{2}(x)=\exp _{a}(x)$, and we set $\rho_{1}(x):=1+x^{2}$ and $\rho_{2}(x):=1+\exp _{2 a}(x)$. 
Let us consider the spaces, for $j=1,2$

$$
C_{\rho_{j}}^{0}(\mathbb{R}):=\left\{f \in C(\mathbb{R}): \lim _{x \rightarrow \pm \infty} \frac{f(x)}{\rho_{j}(x)}=\ell_{f} \in \mathbb{R}\right\} .
$$

Note that if $f \in C_{\rho_{j}}^{0}(\mathbb{R})$, then $|f(x)| \leq M_{f} \rho_{j}(x)$, for a suitable constant $M_{f}>0$ and $x \in \mathbb{R}$. We define a norm on the space $C_{\rho_{j}}^{0}(\mathbb{R})$ on setting

$$
\|f\|_{\rho_{j}}:=\left\|\frac{f}{\rho_{j}}\right\|_{\infty} .
$$

We are ready to prove the main theorem of this section.

Theorem 4.3. Let $j=1,2$ and let $f \in C_{\rho_{j}}^{0}(\mathbb{R})$. Then, under the assumptions of Proposition 4.1 and Corollary 4.2, we have

$$
\lim _{n \rightarrow+\infty}\left\|T_{n} f-f\right\|_{\rho_{j}}=0 .
$$

Proof. First, consider the case $j=1$. The test functions $e_{j}$ obviously belong to $C_{\rho_{1}}^{0}(\mathbb{R})$ and moreover

$$
\left\|\frac{T_{n} e_{0}-e_{0}}{\rho_{1}}\right\|_{\infty}=\left\|\frac{A_{a, n} A_{-a, n}-1}{\rho_{1}}\right\|_{\infty} \leq\left|A_{a, n} A_{-a, n}-1\right|,
$$

and the last term tends to 0 as $n \rightarrow+\infty$. Next,

$$
\begin{aligned}
\left\|\frac{T_{n} e_{1}-e_{1}}{\rho_{1}}\right\|_{\infty} \leq & \left|A_{a, n} A_{-a, n}-1\right| \sup _{x \in \mathbb{R}} \frac{|x|}{x^{2}+1} \\
& +\frac{1}{a} A_{a, n} A_{-a, n}\left|\log A_{a, n}\right|+A_{a, n}\left|B_{-a, n}\right|,
\end{aligned}
$$

and so

$$
\lim _{n \rightarrow+\infty}\left\|\frac{T_{n} e_{1}-e_{1}}{\rho_{1}}\right\|_{\infty}=0 .
$$

Analogously, one can see that

$$
\lim _{n \rightarrow+\infty}\left\|\frac{T_{n} e_{2}-e_{2}}{\rho_{1}}\right\|_{\infty}=0 .
$$

For $j=2$, taking into account of (4.2) and Proposition 3.1, we obtain again the convergence on the test functions $\exp ^{k}(a x)$, for $k=0,1,2$. Applying Theorem 2 in [14] we obtain the assertion

\section{Quantitative Estimates}

For $K_{n} \in L_{\exp _{a}}^{*}(\mathbb{R})$, we define the absolute exponential moment of order 1 of $K_{n}$ as

$$
\widetilde{B}_{-a, n}:=\int_{-\infty}^{\infty} e^{-a t}|t| K_{n}(t) \mathrm{d} t
$$


In the spaces $C_{\rho_{j}}^{0}(\mathbb{R}), j=1,2$ we can define various modulus of continuity. We begin with an estimate of the convergence expressed by Theorem 4.3, in terms of the classical modulus of continuity $\omega$ defined by

$$
\omega(f, \delta):=\sup _{|h| \leq \delta}|f(x+h)-f(x)| \quad(\delta>0) .
$$

As it is well-known, for any uniformly continuous function $f$ one has $\lim _{\delta \rightarrow 0} \omega(f, \delta)=0$. We have the following estimate

Theorem 5.1. Let $K_{n} \in L_{\exp _{a}}^{*}(\mathbb{R})$, for sufficiently large values of $n \in \mathbb{N}$, and let $f \in C_{\rho_{j}}^{0}(\mathbb{R})$. Then for every $\delta>0$, we have

$$
\begin{aligned}
\left\|T_{n} f-f\right\|_{\rho_{j}} \leq & \omega(f, \delta) A_{a, n}\left(A_{-a, n}+\frac{1}{\delta} \widetilde{B}_{-a, n}+\frac{1}{a \delta} A_{-a, n}\left|\log A_{a, n}\right|\right) \\
& +\|f\|_{\rho_{j}}\left|A_{a, n} A_{-a, n}-1\right| .
\end{aligned}
$$

Proof. For every $x \in \mathbb{R}$ and $\delta>0$ we have

$$
\begin{aligned}
\left|\left(T_{n} f\right)(x)-f(x)\right| \leq & A_{a, n} \int_{-\infty}^{\infty} e^{-a t} \omega\left(f,\left|t-\frac{1}{a} \log A_{a, n}\right|\right) K_{n}(t) \mathrm{d} t \\
& +|f(x)|\left|A_{a, n} A_{-a, n}-1\right|=: I_{1}+I_{2} .
\end{aligned}
$$

Thus we have to estimate only $I_{1}$. In order to do that, we use the following well-known property of $\omega$ (see e.g. [4])

$$
\begin{gathered}
\omega(f, \lambda \delta) \leq(1+\lambda) \omega(f, \delta) \quad(\lambda, \delta>0) . \\
\text { Setting } \lambda:=\frac{\left|t-\frac{1}{a} \log A_{a, n}\right|}{\delta}, \text { we have } \\
I_{1} \leq A_{a, n} \omega(f, \delta) \int_{-\infty}^{\infty} e^{-a t}\left(1+\frac{\left|t-\frac{1}{a} \log A_{a, n}\right|}{\delta}\right) K_{n}(t) \mathrm{d} t \\
\leq \omega(f, \delta) A_{a, n}\left(A_{-a, n}+\frac{1}{\delta} \int_{-\infty}^{\infty} e^{-a t}\left(|t|+a^{-1}\left|\log A_{a, n}\right|\right) K_{n}(t) \mathrm{d} t\right) \\
\leq \omega(f, \delta) A_{a, n}\left(A_{-a, n}+\frac{1}{\delta} \widetilde{B}_{-a, n}+\frac{1}{a \delta} A_{-a, n}\left|\log A_{a, n}\right|\right) .
\end{gathered}
$$

Passing to norm, we get the desired result

$$
\begin{aligned}
\left\|T_{n} f-f\right\|_{\rho_{j}} \leq & \omega(f, \delta) A_{a, n}\left(A_{-a, n}+\frac{1}{\delta} \widetilde{B}_{-a, n}+\frac{1}{a \delta} A_{-a, n}\left|\log A_{a, n}\right|\right) \\
& +\|f\|_{\rho_{j}}\left|A_{a, n} A_{-a, n}-1\right|
\end{aligned}
$$

Corollary 5.2. Let the assumptions of Theorem 5.1 be satisfied, and (4.1) holds. If moreover there is $\alpha>0$ such that $\left(n^{\alpha} \widetilde{B}_{-a, n}\right)_{n}$, and $\left(n^{\alpha}\left|\log A_{a, n}\right|\right)_{n}$ are bounded sequences, then there exists an absolute constant $M>0$ such that

$$
\left\|T_{n} f-f\right\|_{\rho_{j}} \leq M \omega\left(f, \frac{1}{n^{\alpha}}\right)+\left|A_{a, n} A_{-a, n}-1\right|\|f\|_{\rho_{j}},
$$

for sufficiently large values of $n \in \mathbb{N}$. 
Proof. It is a consequence of Theorem 5.1 on setting $\delta=n^{-\alpha}$

Remark 5.3. Note that, under the assumptions of Corollary 5.2, if the function $f$ satisfies a Lipschitz condition of order 1 , and $\left|A_{a, n} A_{-a, n}-1\right|=\mathcal{O}\left(n^{-\alpha}\right)$ as $n \rightarrow+\infty$, then

$$
\left\|T_{n} f-f\right\|_{\rho_{j}}=\mathcal{O}\left(n^{-\alpha}\right) \quad(n \rightarrow+\infty) .
$$

Now we state another estimate using a suitable weighted modulus of continuity. In order to do that, we introduce the exponential moment of order 4 of $K_{n} \in L_{\exp _{a}}^{*}(\mathbb{R})$ setting

$$
E_{-a, n}:=\int_{-\infty}^{\infty} e^{-a t} t^{4} K_{n}(t) \mathrm{d} t .
$$

In order to establish rate of convergence, we will use special kind of modulus of continuity $\widetilde{\omega}$ which is compatible with the space $C_{\rho_{1}}^{0}(\mathbb{R})$. This weighted modulus of continuity was first introduced in [27] for $f \in C_{\rho_{1}}^{0}\left(\mathbb{R}_{0}^{+}\right)$, then considered in [5] for $f \in C_{\rho_{1}}^{0}(\mathbb{R})$ as follows:

$$
\widetilde{\omega}(f, \delta)=\sup _{x \in \mathbb{R},|h| \leq \delta} \frac{|f(x+h)-f(x)|}{1+(h+x)^{2}} .
$$

For any function $f \in C_{\rho_{1}}^{0}(\mathbb{R}), m \in \mathbb{N}$ and $\lambda, \delta \in \mathbb{R}^{+}, \widetilde{\omega}$ has the following properties (see $[5,27])$ :

1. $\widetilde{\omega}(f, \delta)$ is a monotonically increasing function of $\delta$,

2. $\lim _{\delta \rightarrow 0^{+}} \widetilde{\omega}(f, \delta)=0$,

3. $\widetilde{\omega}(f, m \delta) \leq m \widetilde{\omega}(f, \delta)$, for every $m \in \mathbb{N}$,

4. $\widetilde{\omega}(f, \lambda \delta) \leq(1+\lambda) \widetilde{\omega}(f, \delta)$ for every $\lambda>0$.

Theorem 5.4. Let $K_{n} \in L_{\exp _{a}}^{*}$ be such that $E_{-a, n}$ exists finite. For $f \in$ $C_{\rho_{1}}^{0}(\mathbb{R})$, there holds

$$
\begin{aligned}
\| T_{n} f & -f \|_{\rho_{1}} \\
\leq & 16 A_{a, n} \widetilde{\omega}\left(f, \sqrt{C_{-a, n}}\right) \\
& \times\left\{C_{-a, n}+\left(1+C_{-a, n}\right) A_{-a, n}+\sqrt{E_{-a, n}+A_{-a, n}\left(1+C_{-a, n}^{2}\right)}\right\} \\
& +\|f\|_{\rho_{1}}\left|A_{a, n} A_{-a, n}-1\right|
\end{aligned}
$$

provided that $\left|a^{-1} \log A_{a, n}\right| \leq \sqrt{C_{-a, n}}$ for sufficiently large $n \in \mathbb{N}$.

Proof. In view of Proposition 4.1, we have

$$
\begin{aligned}
\left|\left(T_{n} f\right)(x)-f(x)\right| \leq & A_{a, n}\left|\int_{-\infty}^{\infty} e^{-a t} f\left(\lambda_{n}(x)+t\right) K_{n}(t) \mathrm{d} t-f(x) \int_{-\infty}^{\infty} e^{-a t} K_{n}(t) \mathrm{d} t\right| \\
& +|f(x)|\left|\left(T_{n} e_{0}\right)(x)-1\right| \\
= & A_{a, n}\left|\int_{-\infty}^{\infty}\left[f\left(\lambda_{n}(x)+t\right)-f(x)\right] e^{-a t} K_{n}(t) \mathrm{d} t\right| \\
& +|f(x)|\left|A_{a, n} A_{-a, n}-1\right| .
\end{aligned}
$$

For any $\delta>0$, properties of $\widetilde{\omega}$ enable us to write

$$
\left|f\left(\lambda_{n}(x)+t\right)-f(x)\right|=\left|f\left(x+t-a^{-1} \log A_{a, n}\right)-f(x)\right|
$$




$$
\begin{aligned}
& \leq\left(1+\left(x+t-a^{-1} \log A_{a, n}\right)^{2}\right) \widetilde{\omega}\left(f,\left|t-a^{-1} \log A_{a, n}\right|\right) \\
& \leq\left(1+\left(x+t-a^{-1} \log A_{a, n}\right)^{2}\right)\left(1+\frac{\left|t-a^{-1} \log A_{a, n}\right|}{\delta}\right) \widetilde{\omega}(f, \delta) .
\end{aligned}
$$

In view of this observation, there holds

$$
\begin{aligned}
& \left|\left(T_{n} f\right)(x)-f(x)\right| \leq \widetilde{\omega}(f, \delta) A_{a, n} \\
& \quad \times \int_{-\infty}^{\infty}\left(1+\left(x+t-a^{-1} \log A_{a, n}\right)^{2}\right)\left(1+\frac{\left|t-a^{-1} \log A_{a, n}\right|}{\delta}\right) e^{-a t} K_{n}(t) \mathrm{d} t \\
& \quad+|f(x)|\left|A_{a, n} A_{-a, n}-1\right| \leq \widetilde{\omega}(f, \delta) A_{a, n} \\
& \quad \times \int_{-\infty}^{\infty}\left(1+\left(x+t-\frac{1}{a} \log A_{a, n}\right)^{2}\right)\left(1+\frac{\left|a^{-1} \log A_{a, n}\right|}{\delta}\right) e^{-a t} K_{n}(t) \mathrm{d} t \\
& \quad+\widetilde{\omega}(f, \delta) A_{a, n} \int_{-\infty}^{\infty}\left(1+\left(x+t-\frac{1}{a} \log A_{a, n}\right)^{2}\right) \frac{|t|}{\delta} e^{-a t} K_{n}(t) \mathrm{d} t \\
& \quad+|f(x)|\left|A_{a, n} A_{-a, n}-1\right|:=J_{1}+J_{2}+|f(x)|\left|A_{a, n} A_{-a, n}-1\right| .
\end{aligned}
$$

For $J_{1}$, using the well-known inequality $|a-b|^{p} \leq 2^{p-1}\left(|a|^{p}+|b|^{p}\right)$, $a, b \in \mathbb{R}, p \geq 1$, we have

$$
\begin{aligned}
J_{1} \leq & \widetilde{\omega}(f, \delta) A_{a, n} \\
& \times\left(1+\frac{\left|a^{-1} \log A_{a, n}\right|}{\delta}\right)\left\{A_{-a, n}+2 C_{-a, n}+2\left(x-a^{-1} \log A_{a, n}\right)^{2} A_{-a, n}\right\} \\
\leq & 4 A_{a, n} \widetilde{\omega}(f, \delta)\left(1+\frac{\left|a^{-1} \log A_{a, n}\right|}{\delta}\right)\left\{C_{-a, n}+\left(1+x^{2}+a^{-2} \log ^{2} A_{a, n}\right) A_{-a, n}\right\} .
\end{aligned}
$$

By the Cauchy-Schwarz inequality for $J_{2}$, we have

$$
\begin{aligned}
J_{2} & =\widetilde{\omega}(f, \delta) A_{a, n} \int_{-\infty}^{\infty}\left(1+\left(x+t-a^{-1} \log A_{a, n}\right)^{2}\right) \frac{|t|}{\delta} e^{-a t} K_{n}(t) \mathrm{d} t \\
& \left.\leq \widetilde{\omega}(f, \delta) A_{a, n} \int_{-\infty}^{\infty}\left(1+\left(x+t-a^{-1} \log A_{a, n}\right)^{2}\right)^{2} e^{-a t} K_{n}(t) \mathrm{d} t\right)^{1 / 2} \frac{\left(C_{-a, n}\right)^{1 / 2}}{\delta} .
\end{aligned}
$$

Repeated application of the inequality $|a-b|^{p} \leq 2^{p-1}\left(|a|^{p}+|b|^{p}\right)$, $a, b \in \mathbb{R}, p \geq 1$ yields

$$
\begin{aligned}
(1 & \left.+\left(x+t-a^{-1} \log A_{a, n}\right)^{2}\right)^{2} \leq\left(1+2\left(t^{2}+\lambda_{n}^{2}(x)\right)\right)^{2} \\
& \leq 2\left(1+4\left(t^{2}+\lambda_{n}^{2}(x)\right)^{2}\right) \leq 2+16\left(t^{4}+\lambda_{n}^{4}(x)\right) \\
& =2+16 t^{4}+16\left(x-a^{-1} \log A_{a, n}\right)^{4} \\
& \leq 2+16 t^{4}+128\left(x^{4}+a^{-4} \log ^{4} A_{a, n}\right) .
\end{aligned}
$$

Using above inequality, we have

$$
J_{2} \leq 16 \widetilde{\omega}(f, \delta) A_{a, n}\left(E_{-a, n}+A_{-a, n}\left(1+x^{4}+a^{-4} \log ^{4} A_{a, n}\right)\right)^{1 / 2} \frac{\left(C_{-a, n}\right)^{1 / 2}}{\delta} .
$$

Collecting all inequalities we get

$$
\left|\left(T_{n} f\right)(x)-f(x)\right|
$$




$$
\begin{aligned}
\leq & 4 A_{a, n} \widetilde{\omega}(f, \delta)\left(1+\frac{\left|a^{-1} \log A_{a, n}\right|}{\delta}\right)\left\{C_{-a, n}+\left(1+x^{2}+a^{-2} \log ^{2} A_{a, n}\right) A_{-a, n}\right\} \\
& +16 \widetilde{\omega}(f, \delta) A_{a, n}\left(E_{-a, n}+A_{-a, n}\left(1+x^{4}+a^{-4} \log ^{4} A_{a, n}\right)\right)^{1 / 2} \frac{\left(C_{-a, n}\right)^{1 / 2}}{\delta} \\
& +|f(x)|\left|A_{a, n} A_{-a, n}-1\right| .
\end{aligned}
$$

Choosing $\delta=\left(C_{-a, n}\right)^{1 / 2}$ with $\left|a^{-1} \log A_{a, n}\right| \leq \delta$ for sufficiently large $n$, dividing both sides by $1+x^{2}$ and taking supremum over all $x$, we obtain

$$
\begin{aligned}
\| T_{n} f & -f \|_{\rho_{1}} \\
\leq & 16 A_{a, n} \tilde{\omega}\left(f, \sqrt{C_{-a, n}}\right)\left\{C_{-a, n}+\left(1+a^{-2} \log ^{2} A_{a, n}\right) A_{-a, n}\right. \\
& \left.+\sqrt{E_{-a, n}+A_{-a, n}\left(1+a^{-4} \log ^{4} A_{a, n}\right)}\right\}+|f(x)|\left|A_{a, n} A_{-a, n}-1\right| \\
\leq & 16 A_{a, n} \tilde{\omega}\left(f, \sqrt{C_{-a, n}}\right)\left\{C_{-a, n}+\left(1+C_{-a, n}\right) A_{-a, n}\right. \\
& \left.+\sqrt{E_{-a, n}+A_{-a, n}\left(1+\left(C_{-a, n}\right)^{2}\right)}\right\} \\
& +\|f\|_{\rho_{1}}\left|A_{a, n} A_{-a, n}-1\right|
\end{aligned}
$$

that is the assertion

Remark 5.5. The estimate of Theorem 5.4 gives an evaluation of the convergence in terms of the modulus of continuity with parameter $\sqrt{C_{-a, n}}$, under the further assumptions that $C_{-a, n} \rightarrow 0$ and $\left|A_{a, n} A_{-a, n}-1\right| \rightarrow 0$ as $n \rightarrow+\infty$. Thus one can obtain a corresponding corollary, as for Theorem 5.1 (see Corollary 5.2 and Remark 5.3).

\section{An Asymptotic Formula}

Here we establish two asymptotic formulae of Voronovskaya type for functions $f \in C_{\rho_{j}}^{0}(\mathbb{R}), j=1,2$, which are locally of class $C^{2}$ at a point $x$. These kinds of formulae give an exact evaluation of the order of pointwise convergence. We examine the case $j=1$.

Theorem 6.1. Let $f \in C_{\rho_{1}}^{0}(\mathbb{R})$ be locally of class $C^{2}$ at a point $x \in \mathbb{R}$. Let $K_{n} \in L_{\exp _{a}}^{*}(\mathbb{R})$, for sufficiently large values of $n \in \mathbb{N}$, be a kernel satisfying the assumptions of Corollary 4.2. Assume that there exist $\alpha>0$ such that the kernel $\left\{K_{n}\right\}$ satisfies further the following conditions:

(i) $\lim _{n \rightarrow+\infty} n^{\alpha}\left(A_{a, n} A_{-a, n}-1\right)=\ell_{0}, \lim _{n \rightarrow+\infty} n^{\alpha} B_{-a, n}=\ell_{1}$, $\lim _{n \rightarrow+\infty} n^{\alpha} C_{-a, n}=\ell_{2}$ and $\lim _{n \rightarrow+\infty} n^{\alpha} \frac{1}{a} \log A_{a, n}=\ell_{3}$, with $\ell_{j} \in \mathbb{R}$, for $j=0,1,2,3$.

(ii) For every $\eta>0$,

$$
\lim _{n \rightarrow+\infty} n^{\alpha} \int_{|t| \geq \eta} e^{-a t}\left(t-\frac{1}{a} \log A_{a, n}\right)^{2} K_{n}(t) \mathrm{d} t=0 .
$$


Then,

$$
\lim _{n \rightarrow+\infty} n^{\alpha}\left[\left(T_{n} f\right)(x)-f(x)\right]=\ell_{0} f(x)+\left(\ell_{1}-\ell_{3}\right) f^{\prime}(x)+\frac{\ell_{2}}{2} f^{\prime \prime}(x) .
$$

Proof. Since $f$ has a polynomial growth of order 2 , and locally of class $C^{2}$ at the point $x$, using a local Taylor formula of the second order, we can write

$$
\begin{aligned}
f\left(\lambda_{n}(x)+t\right)= & f\left(x-\frac{1}{a} \log A_{a, n}+t\right)=f(x)+f^{\prime}(x)\left(t-\frac{1}{a} \log A_{a, n}\right) \\
& +\frac{f^{\prime \prime}(x)}{2}\left(t-\frac{1}{a} \log A_{a, n}\right)^{2}+r_{x}\left(t-\frac{1}{a} \log A_{a, n}\right) \\
& \times\left(t-\frac{1}{a} \log A_{a, n}\right)^{2}
\end{aligned}
$$

where $r_{x}(y)$ is a bounded function such that $\lim _{y \rightarrow 0} r_{x}(y)=0$.

We write

$$
\begin{aligned}
\left(T_{n} f\right)(x)-f(x)= & A_{a, n} \int_{-\infty}^{\infty} e^{-a t}\left[f\left(\lambda_{n}(x)+t\right)-f(x)\right] K_{n}(t) \mathrm{d} t \\
& +\left(A_{a, n} A_{-a, n}-1\right) f(x)=: I_{1}+I_{2} .
\end{aligned}
$$

As to $I_{2}$ we have by assumptions that $n^{\alpha} I_{2} \rightarrow \ell_{0} f(x)$ as $n \rightarrow+\infty$. Therefore we evaluate now the term $I_{1}$. Inserting (6.2) in $I_{1}$, we can write

$$
\begin{aligned}
I_{1}= & A_{a, n}\left(B_{-a, n}-A_{-a, n} \frac{1}{a} \log A_{a, n}\right) f^{\prime}(x) \\
& +A_{a, n}\left(C_{-a, n}+\frac{A_{-a, n}}{a^{2}} \log ^{2} A_{a, n}-\frac{2}{a} B_{-a, n} \log A_{a, n}\right) \frac{f^{\prime \prime}(x)}{2}+R_{x} \\
= & : I_{1}^{1}+I_{1}^{2}+R_{x},
\end{aligned}
$$

where

$$
R_{x}:=A_{a, n} \int_{-\infty}^{\infty} e^{-a t} r_{x}\left(t-\frac{1}{a} \log A_{a, n}\right)\left(t-\frac{1}{a} \log A_{a, n}\right)^{2} K_{n}(t) \mathrm{d} t .
$$

Now,

$$
\lim _{n \rightarrow+\infty} n^{\alpha} I_{1}^{1}=\left(\ell_{1}-\ell_{3}\right) f^{\prime}(x), \quad \lim _{n \rightarrow+\infty} n^{\alpha} I_{1}^{2}=\frac{\ell_{2}}{2} f^{\prime \prime}(x) .
$$

Thus we have to estimate the remainder term $R_{x}$.

Since $\lim _{y \rightarrow 0} r_{x}(y)=0$, given an arbitrary $\varepsilon>0$, there is $\left.\delta \in\right] 0,1[$ such that $\left|r_{x}(y)\right|<\varepsilon$ whenever $|y| \leq \delta$. We take now an index $\bar{n}$ such that for $n \geq \bar{n}$, one has $a^{-1}\left|\log A_{a, n}\right|<\delta / 2$. Thus for $|t|<\delta / 2$, we have also $\left|t-a^{-1} \log A_{a, n}\right|<\delta$. Thus

$$
\left|r_{x}\left(t-\frac{1}{a} \log A_{a, n}\right)\right|<\varepsilon \quad(|t|<\delta / 2) .
$$

Writing

$$
\begin{aligned}
R_{x} & =A_{a, n}\left\{\int_{-\delta / 2}^{\delta / 2}+\int_{|t| \geq \delta / 2}\right\} e^{-a t} r_{x}\left(t-\frac{1}{a} \log A_{a, n}\right)\left(\left(t-\frac{1}{a} \log A_{a, n}\right)^{2} K_{n}(t) \mathrm{d} t\right. \\
& =: R_{1}+R_{2},
\end{aligned}
$$


we have, by (6.4),

$$
\left|R_{1}\right| \leq A_{a, n} \varepsilon\left(C_{-a, n}+A_{-a, n} \frac{1}{a^{2}} \log ^{2} A_{a, n}-\frac{2 B_{-a, n}}{a} \log A_{a, n}\right) .
$$

Therefore using the assumptions (i) we have, for a suitable absolute positive constant $M$,

$$
\limsup _{n \rightarrow+\infty} n^{\alpha}\left|R_{1}\right| \leq M \varepsilon
$$

As to the term $R_{2}$, using the boundedness of $r_{x}$ we can write

$$
\left|R_{2}\right| \leq A_{a, n}\left\|r_{x}\right\|_{\infty} \int_{|t| \geq \delta / 2} e^{-a t}\left(t-\frac{1}{a} \log A_{a, n}\right)^{2} K_{n}(t) \mathrm{d} t,
$$

and by (ii) we obtain

$$
\lim _{n \rightarrow+\infty} n^{\alpha}\left|R_{2}\right|=0
$$

This implies

$$
\limsup _{n \rightarrow+\infty} n^{\alpha}\left|R_{x}\right| \leq M \varepsilon
$$

that is $\left|R_{x}\right| \rightarrow 0$ as $n \rightarrow+\infty$. Thus the theorem is completely proved

Theorem 5.4 works perfectly in several particular examples, as we will see in the next section. But in certain situations, as for example, the momenttype operators, formula (6.1) becomes

$$
\lim _{n \rightarrow+\infty} n^{\alpha}\left[\left(T_{n} f\right)(x)-f(x)\right]=0,
$$

for a suitable constant $\alpha$ for which all the assumptions are satisfied. In case of the moment-type operator, we have $\alpha=1$. If we try to take $\alpha=2$ some of the assumptions (i) of Theorem 5.4 are not satisfied. This result is however interesting, but it gives no exact information about the pointwise order of approximation at a point $x$. Therefore, we will formulate a slight generalization of the above theorem, in order to include also the case of moment-type operators, by changing assumptions (i). We have the following

Theorem 6.2. Let $f \in C_{\rho_{1}}^{0}(\mathbb{R})$ be locally of class $C^{2}$ at a point $x \in \mathbb{R}$. Let $K_{n} \in L_{\exp _{a}}^{*}(\mathbb{R})$, for sufficiently large values of $n \in \mathbb{N}$, be a kernel satisfying the assumptions of Corollary 4.2. Assume that there exist $\alpha>0$ such that the kernel $\left\{K_{n}\right\}$ satisfies assumption (ii) of Theorem 5.4 and the following conditions:

(j) $\lim _{n \rightarrow+\infty} n^{\alpha}\left(A_{a, n} A_{-a, n}-1\right)=\ell_{0}$, $\lim _{n \rightarrow+\infty} n^{\alpha}\left(B_{-a, n}-A_{-a, n} \frac{1}{a} \log A_{a, n}\right)=\lambda_{1}$,

(jj) $\lim _{n \rightarrow+\infty} n^{\alpha}\left(C_{-a, n}+\frac{A_{-a, n}}{a^{2}} \log ^{2} A_{a, n}-\frac{2}{a} B_{-a, n} \log A_{a, n}\right)=\lambda_{2}$

Then,

$$
\lim _{n \rightarrow+\infty} n^{\alpha}\left[\left(T_{n} f\right)(x)-f(x)\right]=\ell_{0} f(x)+\lambda_{1} f^{\prime}(x)+\frac{\lambda_{2}}{2} f^{\prime \prime}(x) .
$$

Proof. The proof is clearly exactly the same. 


\section{Examples}

In this section we discuss some examples of kernels $\left\{K_{n}\right\}$ for which the theory developed can be applied.

(1) The Gauss-Weierstrass kernel

For $n \in \mathbb{N}$, let us consider the kernel $\left\{K_{n}\right\}$ with

$$
K_{n}(t)=\sqrt{\frac{n}{\pi}} e^{-n t^{2}} \quad(t \in \mathbb{R}) .
$$

This kernel is defined by even functions. First we evaluate the exponential moments of orders $0,1,2$. In order to do that, we first calculate the coefficients $A_{a, n}$ using a differentiation under the integral. By solving a simple first order linear differential equation, we obtain

$$
A_{a, n}=A_{-a, n}=\sqrt{\frac{n}{\pi}} \int_{-\infty}^{\infty} e^{a t} e^{-n t^{2}} \mathrm{~d} t=e^{a^{2} /(4 n)} .
$$

Hence $A_{a, n} A_{-a, n}=e^{a^{2} /(2 n)}$. Taking $\alpha=1$, we obtain

$$
\lim _{n \rightarrow+\infty} n\left(A_{a, n} A_{-a, n}-1\right)=\lim _{n \rightarrow+\infty} n\left(e^{a^{2} /(2 n)}-1\right)=\frac{a^{2}}{2} .
$$

Moreover, using partial integrations, one has

$$
B_{-a, n}=-\frac{a}{2 n} e^{a^{2} / 4 n}, C_{-a, n}=\frac{1}{2 n} A_{a, n}+\frac{a^{2}}{4 n^{2}} A_{a, n},
$$

and so

$$
\lim _{n \rightarrow+\infty} n B_{-a, n}=\frac{1}{2}, \lim _{n \rightarrow+\infty} n C_{-a, n}=\frac{1}{2} .
$$

Next, we have

$$
\lim _{n \rightarrow+\infty} n \log A_{a, n}=\frac{a^{2}}{4},
$$

therefore we have also

$$
\lim _{n \rightarrow+\infty} n \log ^{2} A_{a, n}=0 .
$$

Therefore, $\ell_{1}-\ell_{3}=-3 a / 4, \ell_{2}=1 / 2$. Now we prove that the GaussWeierstrass kernel satisfies also assumption (ii) of Theorem 5.4. Let $\eta>0$ be fixed. Let us set

$$
J:=n \int_{|t| \geq \eta} e^{-a t}\left(t-\frac{1}{a} \log A_{a, n}\right)^{2} K_{n}(t) \mathrm{d} t .
$$

Then

$$
\begin{aligned}
J \leq & n \int_{|t| \geq \eta} e^{-a t} t^{2} K_{n}(t) \mathrm{d} t+n\left(\frac{1}{a^{2}} \log ^{2} A_{a, n}\right) \int_{|t| \geq \eta} e^{-a t} K_{n}(t) \mathrm{d} t \\
& +2 n \frac{1}{a}\left|\log A_{a, n}\right| \int_{|t| \geq \eta} e^{-a t}|t| K_{n}(t) \mathrm{d} t=: J_{1}+J_{2}+J_{3} .
\end{aligned}
$$


As to $J_{1}$ we can write

$$
\begin{aligned}
J_{1} & =\frac{n \sqrt{n}}{\sqrt{\pi}} \int_{|t| \geq \eta} e^{-a t} t^{2} e^{-n t^{2}} \mathrm{~d} t \\
& =\frac{n \sqrt{n}}{\sqrt{\pi}}\left\{\int_{\eta}^{\infty} e^{-a t} t^{2} e^{-n t^{2}} \mathrm{~d} t+\int_{\eta}^{\infty} e^{a t} t^{2} e^{-n t^{2}} \mathrm{~d} t\right\} \\
& =: J_{1}^{1}+J_{1}^{2} .
\end{aligned}
$$

For $J_{1}^{1}$ we have by a suitable substitution,

$$
J_{1}^{1} \leq \frac{e^{-a \eta}}{\sqrt{\pi}} \int_{\sqrt{n} \eta}^{\infty} v^{2} e^{-v^{2}} d v
$$

and so by the absolute continuity of the Lebesgue integral, we obtain

$$
\lim _{n \rightarrow+\infty} J_{1}^{1}=0 \text {. }
$$

Analogously,

$$
J_{1}^{2} \leq \frac{1}{\sqrt{\pi}} \int_{\sqrt{n} \eta}^{\infty} e^{a v} v^{2} e^{-v^{2}} d v,
$$

and again, since the integrand in the right-hand side is Lebesgue integrable, we obtain $J_{1}^{2} \rightarrow 0$ as $n \rightarrow+\infty$. Next, we evaluate $J_{2}$. We have immediately

$$
J_{2} \leq \frac{n}{a^{2}} \log ^{2} A_{a, n} A_{-a, n} \rightarrow 0, \quad(n \rightarrow+\infty) .
$$

Finally, we evaluate $J_{3}$. Using the same reasonings as for the estimate of $J_{1}$, we have

$$
\begin{aligned}
J_{3} & \leq \frac{a \sqrt{n}}{2 \sqrt{\pi}}\left\{e^{-a \eta} \int_{\eta}^{\infty} t e^{-n t^{2}} \mathrm{~d} t+\int_{\eta}^{\infty} e^{a t} t e^{-n t^{2}} \mathrm{~d} t\right\} \\
& =: J_{3}^{1}+J_{3}^{2} .
\end{aligned}
$$

As for $J_{1}$ we obtain easily that $J_{3}^{1} \rightarrow 0, J_{3}^{2} \rightarrow 0$ as $n \rightarrow+\infty$. Concluding, we obtain assumption (ii) of Theorem 5.4. Therefore all the assumptions introduced are satisfied with $\alpha=1$. The asymptotic formula of Theorem 5.4 reads

$$
\lim _{n \rightarrow+\infty} n\left(\left(T_{n} f\right)(x)-f(x)\right)=\frac{a^{2}}{2} f(x)-\frac{3 a}{4} f^{\prime}(x)+\frac{1}{4} f^{\prime \prime}(x),
$$

which is a result of [26].

(2) The Picard Kernel

For $n \in \mathbb{N}$, let us consider the kernel $\left\{K_{n}\right\}$ with

$$
K_{n}(t)=\frac{\sqrt{n}}{2} e^{-\sqrt{n}|t|} \quad(t \in \mathbb{R}) .
$$

Also this kernel is defined by even functions. Let us evaluate the exponential moments of order $0,1,2$. First we have

$$
A_{a, n}=A_{-a, n}=\frac{n}{n-a^{2}}, \text { and } A_{a, n}^{2}=\left(\frac{n}{n-a^{2}}\right)^{2}
$$


and from sufficiently large values of $n, A_{a, n}$ is positive. Moreover

$$
\lim _{n \rightarrow+\infty} n\left(A_{a, n} A_{-a, n}-1\right)=2 a^{2}, \lim _{n \rightarrow+\infty} n \log A_{a, n}=a^{2} .
$$

Now, we evaluate the moment $B_{-a, n}$. Using elementary calculation, based on partial integration, we can see that

$$
B_{-a, n}=-\frac{2 a n}{\left(n-a^{2}\right)^{2}}, C_{-a, n}=\sqrt{n}\left(\frac{1}{(a+\sqrt{n})^{3}}+\frac{1}{(\sqrt{n}-a)^{3}}\right) .
$$

Therefore,

$$
\lim _{n \rightarrow+\infty} n B_{-a, n}=-2 a, \lim _{n \rightarrow+\infty} n C_{-a, n}=2 .
$$

Finally we check assumption (ii) of Theorem 5.4. We proceed as in the previous example. Let $\eta>0$ be fixed and set

$$
J:=n \int_{|t| \geq \eta} e^{-a t}\left(t-\frac{1}{a} \log A_{a, n}\right)^{2} K_{n}(t) \mathrm{d} t .
$$

We have

$$
\begin{aligned}
J \leq & \frac{n \sqrt{n}}{2}\left\{\int_{|t| \geq \eta} e^{-a t} t^{2} e^{-\sqrt{n}|t|} \mathrm{d} t+\frac{1}{a^{2}} \log ^{2} \frac{n}{n-a^{2}} \int_{|t| \geq \eta} e^{-a t} e^{-\sqrt{n}|t|} \mathrm{d} t\right. \\
& \left.+\frac{2}{a} \log \frac{n}{n-a^{2}} \int_{|t| \geq \eta} e^{-a t}|t| e^{-\sqrt{n}|t|} \mathrm{d} t\right\}=: J_{1}+J_{2}+J_{3} .
\end{aligned}
$$

Using now elementary calculations, based on suitable substitutions, it is easy to see that

$$
J_{1} \leq \frac{e^{-a \eta}}{2} \int_{\sqrt{n} \eta}^{\infty} u^{2} e^{-u / 2} d u+\frac{1}{2} \int_{\sqrt{n} \eta}^{\infty} u^{2} e^{-u / 2} d u
$$

for every $n$ such that $1-a / \sqrt{n}>1 / 2$. Thus, $J_{1} \rightarrow 0$ as $n \rightarrow+\infty$.

Next, let us consider $J_{2}$. We have easily

$$
J_{2} \leq \frac{n}{a^{2}} \log ^{2} \frac{n}{n-a^{2}} A_{-a, n},
$$

and so $J_{2} \rightarrow 0$ as $n \rightarrow+\infty$. Finally, as to $J_{3}$ we have

$$
J_{3} \leq \frac{e^{-a \eta} \sqrt{n}}{a} \log \frac{n}{n-a^{2}} \int_{\sqrt{n} \eta}^{\infty} v e^{-v} d v+\frac{\sqrt{n}}{a} \log \frac{n}{n-a^{2}} \int_{\sqrt{n} \eta}^{\infty} u e^{-u / 2} d u
$$

for every $n$ such that $1-a / \sqrt{n}>1 / 2$. Therefore, $J_{3} \rightarrow 0$ as $n \rightarrow+\infty$, and assumption (ii) is satisfied. Therefore all the assumptions introduced are satisfied with $\alpha=1$. The asymptotic formula of Theorem 5.4 reads

$$
\lim _{n \rightarrow+\infty} n\left(\left(T_{n} f\right)(x)-f(x)\right)=2 a^{2} f(x)-3 a f^{\prime}(x)+f^{\prime \prime}(x)
$$

which is a result of [6]. 
(3) The moment kernel

For $n \in \mathbb{N}$ let us consider the kernel $\left\{K_{n}\right\}$ with

$$
K_{n}(t)=n \chi_{[0,1 / n]}(t) \quad(t \in \mathbb{R}) .
$$

This kernel is not even, and the functions $K_{n}$ have compact support. We calculate now the coefficients $A_{a, n}, A_{-a, n}, B_{-a, n}$ and $C_{-a, n}$ and some their properties. We have

$$
A_{a, n}=n \int_{0}^{1 / n} e^{a t} \mathrm{~d} t=\frac{n}{a}\left(e^{a / n}-1\right), A_{-a, n}=\frac{n}{a}\left(1-e^{-a / n}\right),
$$

and so

$$
\lim _{n \rightarrow+\infty} A_{a, n}=\lim _{n \rightarrow+\infty} A_{-a, n}=1, \quad \lim _{n \rightarrow+\infty} \frac{1}{a} n \log A_{a, n}=\frac{1}{2} .
$$

Moreover,

$$
\lim _{n \rightarrow+\infty} n\left(A_{a, n} A_{-a, n}-1\right)=0 .
$$

Next,

$$
B_{-a, n}=-\frac{e^{-a / n}}{a}+\frac{n}{a^{2}}\left(1-e^{-a / n}\right), C_{-a, n}=-\frac{e^{-a / n}}{a n}+\frac{2}{a} B_{-a, n},
$$

and both tend to zero as $n \rightarrow+\infty$. Now,

$$
\lim _{n \rightarrow+\infty} n B_{-a, n}=1 / 2, \lim _{n \rightarrow+\infty} n C_{a, n}=0 .
$$

Concerning assumption (ii) of Theorem 5.4, since the functions $K_{n}$ have supports $[0,1 / n]$ for any given $\eta>0$, one can find an integer $n_{0}$ such that for any $n \geq n_{0}$ the set $\{t:|t| \geq \eta\}$ does not intersect the interval $[0,1 / n]$, and so denoting $H_{n, \eta}:=\{t:|t| \geq \eta\} \cap[0,1 / n]$, we have

$$
\int_{H_{n}} e^{-a t}\left(t-\frac{1}{a} \log A_{a, n}\right)^{2} \mathrm{~d} t=0
$$

This implies that assumption (ii) is trivially satisfied. Therefore all the assumptions introduced are satisfied with $\alpha=1$. The asymptotic formula of Theorem 5.4 reads

$$
\lim _{n \rightarrow+\infty} n\left(\left(T_{n} f\right)(x)-f(x)\right)=0 .
$$

This result is not fully satisfactory, due to the fact that we have no a precise order of pointwise approximation. Therefore we now employ Theorem 6.2 with $\alpha=2$. In order to do that, we have to check the assumptions $(\mathrm{j})$ and $(\mathrm{jj})$. As to $(\mathrm{j})$, we first calculate the limit

$$
\lim _{n \rightarrow+\infty} n^{2}\left(B_{-a, n}-\frac{1}{a} \log A_{a, n}\right),
$$

which can be written as

$$
\lim _{n \rightarrow+\infty} \frac{n^{2}}{a^{2}}\left(-a e^{-a / n}+a \frac{n}{a}\left(1-e^{-a / n}\right)-a \log \left(\frac{n}{a}\left(e^{a / n}-1\right)\right)\right) .
$$


This can be interpreted as a restriction of the limit

$$
\lim _{x \rightarrow 0} \frac{a}{x^{2}}\left(-e^{-x}+\frac{1-e^{-x}}{x}-\log \left(\frac{e^{x}-1}{x}\right)\right) .
$$

The above limit can be solved using elementary techniques, based on the L'Hospital rule and its value is given by $-3 a / 8$. Now, in order to calculate $\lambda_{1}$ we write

$$
\begin{aligned}
& n^{2}\left(B_{-a, n}-A_{-a, n} \frac{1}{a} \log A_{a, n}\right) \\
& \quad=n^{2}\left(B_{-a, n}-\frac{1}{a} \log A_{a, n}\right)+n^{2} \frac{1}{a} \log A_{a, n}\left(1-A_{-a, n}\right),
\end{aligned}
$$

thus we consider only the limit

$$
\lim _{n \rightarrow+\infty} n^{2} \frac{1}{a} \log A_{a, n}\left(1-A_{-a, n}\right) .
$$

Since as we have seen before $\lim _{n \rightarrow+\infty}(n / a) \log A_{a, n}=1 / 2$, we have to calculate only $\lim _{n \rightarrow+\infty} n\left(1-A_{-a, n}\right)$ and it is not difficult to see that its value is given by $a / 2$. Thus, finally we obtain $\lambda_{1}=-a / 8$.

Now we proceed to the calculation of $\lambda_{2}$. At first we calculate the limit

$$
\lim _{n \rightarrow+\infty} n^{2} C_{-a, n}
$$

Again this limit can be considered as a restriction of the limit

$$
\lim _{x \rightarrow 0} \frac{1}{x^{2}}\left(-x e^{-x}-2 e^{-x}+2 \frac{1-e^{-x}}{x}\right),
$$

and using an analogous elementary approach, its value is given by $1 / 3$.

Next, using the previous results, we have immediately

$$
\lim _{n \rightarrow+\infty} n^{2} \frac{A_{-a, n}}{a^{2}} \log ^{2} A_{a, n}=\frac{1}{4},
$$

and

$$
\lim _{n \rightarrow+\infty} \frac{2}{a} n^{2} B_{-a, n} \log A_{a, n}=1 / 2 .
$$

Thus, we have $\lambda_{2}=1 / 12$.

The last limit $\ell_{0}=\lim _{n \rightarrow+\infty} n^{2}\left(A_{a, n} A_{-a, n}-1\right)$ can be obtained with the same reasonings as a restriction of the limit

$$
\lim _{x \rightarrow 0} a^{2} \frac{e^{x}+e^{-x}-2-x^{2}}{x^{4}}
$$

which is given by $a^{2} / 12$. Concluding, the Voronovskaya formula (6.5) with $\alpha=2$ for the moment type operator is given by (see also [26])

$$
\lim _{n \rightarrow+\infty} n^{2}\left[\left(T_{n} f\right)(x)-f(x)\right]=\frac{a^{2}}{12} f(x)-\frac{a}{8} f^{\prime}(x)+\frac{1}{24} f^{\prime \prime}(x) .
$$


(4) A spline kernel

For $n \in \mathbb{N}$ let us consider the kernel $\left\{K_{n}\right\}$ with

$$
K_{n}(t)=n(1-|n t|) \chi_{[-1 / n, 1 / n]}(t) \quad(t \in \mathbb{R}) .
$$

For this kernel we have, by elementary calculations

$$
A_{a, n}=A_{-a, n}=n \int_{-1 / n}^{1 / n} e^{a t}(1-n|t|) \mathrm{d} t=\frac{2 n^{2}}{a^{2}}(\cosh (a / n)-1),
$$

and so

$$
\lim _{n \rightarrow+\infty} A_{ \pm a, n}=1, \quad \lim _{n \rightarrow+\infty} n^{2}\left(A_{a, n} A_{-a, n}-1\right)=\frac{a^{2}}{6},
$$

and $\lim _{n \rightarrow+\infty} n^{2} \log A_{a, n}=a^{2} / 12$. Next,

$$
B_{-a, n}=n \int_{-1 / n}^{1 / n} t e^{-a t}(1-n|t|) \mathrm{d} t=-\frac{2 n}{a^{2}} \sinh (a / n)+\frac{4 n^{2}}{a^{3}}(\cosh (a / n)-1),
$$

and

$$
\lim _{n \rightarrow+\infty} n^{2} B_{-a, n}=-\frac{a}{6} .
$$

As to $C_{-a, n}$ we have

$$
\begin{aligned}
C_{-a, n} & =n \int_{-1 / n}^{1 / n} t^{2}(1-n|t|) e^{-a t} \mathrm{~d} t \\
& =\frac{2}{a^{2}} \cosh (a / n)-\frac{8 n}{a^{3}} \sinh (a / n)+\frac{12 n^{2}}{a^{4}}(\cosh (a / n)-1),
\end{aligned}
$$

and so

$$
\lim _{n \rightarrow+\infty} n^{2} C_{-a, n}=\frac{1}{6} .
$$

Finally, since the functions $K_{n}$ have compact supports $[-1 / n, 1 / n]$, we easily see, as in the previous example, that (ii) of Theorem 5.4 is satisfied. Thus, all the assumptions used in the previous theory are satisfied with $\alpha=2$. The corresponding asymptotic formula is given by

$$
\lim _{n \rightarrow+\infty} n^{2}\left(\left(T_{n} f\right)(x)-f(x)\right)=\frac{a^{2}}{6} f(x)-\frac{a}{4} f^{\prime}(x)+\frac{1}{12} f^{\prime \prime}(x) .
$$

Remark 7.1. The kernel of Example (4) is generated by the spline function of second order, defined by

$$
\beta_{2}(x)=(1-|x|) \chi_{[-1,1]}(x) \quad(x \in \mathbb{R}) .
$$

The functions $K_{n}$ are given by $K_{n}(x)=n \beta_{2}(n x)$, for $x \in \mathbb{R}$. The spline functions of order $k$ are given by the formula (see e.g. [11,24])

$$
\beta_{k}(x)=\frac{1}{(k-1) !} \sum_{j=0}^{k}(-1)^{j}\left(\begin{array}{l}
k \\
j
\end{array}\right)\left(\frac{k}{2}+x-j\right)_{+}^{k-1},
$$

where for any real number $r, r_{+}$denotes its positive part. The theory may be applied also to any kernel generated by the spline $\beta_{k}$. 
Remark 7.2. Note that the kernels of any of the previous examples satisfy the assumptions of Theorem 5.4, in particular the inequality

$$
\frac{1}{a}\left|\log A_{a, n}\right| \leq \sqrt{C_{-a, n}},
$$

for every $a>0$ and sufficiently large $n \in \mathbb{N}$. First, note that since in any of the above examples $A_{a, n} \geq 1$, we have $\log A_{a, n} \geq 0$. For the modified Gauss-Weierstrass kernel, one has easily

$$
\sqrt{C_{-a, n}}>\frac{a}{2 n}>\frac{1}{a} \log A_{a, n} .
$$

For the modified Picard kernel, one can employ the calculations of the limits in Example (2): since $n C_{-a, n} \rightarrow 2$ as $n \rightarrow+\infty$, and

$$
\lim _{n \rightarrow+\infty} \frac{\sqrt{n}}{a} \log A_{a, n}=0,
$$

for sufficiently large values of $n$ we obtain the assertion. Similar arguments can be used for the remaining examples.

Remark 7.3. Further examples can be obtained using Phillips type operators and Post-Widder type operators which act on functions defined over the positive real axis, (see $[16,18,19]$ in which some modified version are introduced).

Remark 7.4. Our approach may be applied also in case of general exponential functions of the form $a^{-x}$ for $a>1$ as studied in [17] for Baskakov-type operators that act on functions defined over the positive real axis.

\section{Acknowledgements}

We wish to thank very much the referees for their valuable suggestions that improved the presentation of the paper.

Open Access. This article is licensed under a Creative Commons Attribution 4.0 International License, which permits use, sharing, adaptation, distribution and reproduction in any medium or format, as long as you give appropriate credit to the original author(s) and the source, provide a link to the Creative Commons licence, and indicate if changes were made. The images or other third party material in this article are included in the article's Creative Commons licence, unless indicated otherwise in a credit line to the material. If material is not included in the article's Creative Commons licence and your intended use is not permitted by statutory regulation or exceeds the permitted use, you will need to obtain permission directly from the copyright holder. To view a copy of this licence, visit http:// creativecommons.org/licenses/by/4.0/.

Publisher's Note Springer Nature remains neutral with regard to jurisdictional claims in published maps and institutional affiliations. 


\section{References}

[1] Agratini, O., Aral, A., Deniz, E.: On two classes of approximation processes of integral type. Positivity 21(3), 1189-1199 (2017)

[2] Altomare, F.: Korovkin-type theorems and approximation by positive linear operators. Surv. Approx. Theory 5, 9-164 (2010)

[3] Altomare, F., Campiti, M.: Korovkin-type approximation theory and its applications, De Gruyter Studies in Mathematics, vol. 17. W. De Gruyter, Berlin (1994)

[4] Anastassiou, G.A., Gal, S.G.: Approx. Theory. Moduli of Continuity and Global Smoothness Preservation. Birkhauser, Boston (2000)

[5] Anastassiou, G.A., Aral, A.: On Gauss-Weierstrass type integral operators. Demonstr. Math. 43(4), 841-849 (2010)

[6] Aral, A.: On generalized Picard integral operators. In: Advances in Summability and Approximation Theory, pp. 157-168. Springer, Berlin (2018)

[7] Bardaro, C., Mantellini, I.: Voronovskaja-type estimates for Mellin convolution operators. Results Math. 50(1-2), 1-16 (2007)

[8] Bardaro, C., Mantellini, I.: A quantitative Voronovskaya formula for Mellin convolution operators. Mediterr. J. Math. 7(4), 483-501 (2010)

[9] Bardaro, C., Musielak, J., Vinti, G.: Nonlinear integral operators and applications, De Gruyter series in Nonlinear analysis and applications, vol. 9. W. De Gruyter, Berlin (2003)

[10] Bohman, H.: On approximation of continuous and of analytic functions. Arkiv Math. 2(3), 43-56 (1952)

[11] Butzer, P.L., Stens, R.L.: Linear prediction by samples from the past. In: Marks II, R.J. (ed.) Advances Topics in Shannon Sampling and Interpolation Theory, Springer Texts Electrical Eng., pp. 157-183. Springer, New York (1993)

[12] Costarelli, D., Vinti, G.: Voronovskaja type theorems and high-order convergence neural network operators with sigmoidal functions. Mediterr. J. Math. 17, art. $77(2020)$

[13] Costarelli, D., Vinti, G.: Asymptotic expansions and Voronovskaja type theorems for the multivariate neural network operators. Math. Found. Comput. 3(1), 41-50 (2020)

[14] Gadjiev, A.D.: The convergence problem for a sequence of positive linear operators on unbounded sets, and theorems analogous to that ofP.P. Korovkin, (Russian). Dokl. AKad. NAuk SSSR 218(5), 1001-1004 (1974)

[15] Gadjiev, A.D.: Theorems of the type of P.P. Korovkin's Theorems. Mat. Zametki 20(5), 781-786 (1976)

[16] Gupta, V., Agrawal, D.: Convergence by modified Post-Widder operators. RACSAM 113, 1475-1486 (2019)

[17] Gupta, V., Holhos, A.: Approximation with arbitrary order by Baskakovtype operators preserving exponential functions. Bull. Malays. Math. Sci. Soc. (2021). https://doi.org/10.1007/s40840-020-01063-x

[18] Gupta, V., Tachev, G.: On approximation properties of phillips operators preserving exponential functions. Mediterr. J. Math. 14, art.177 (2017)

[19] Gupta, V., Tachev, G.: Approximation with positive linear operators and linear combinations, Series: Developments in Mathematics, vol. 50. Springer, Cham (2017) 
[20] Karsli, H.: Convergence and rate of convergence by nonlinear singular operators depending on two parameters. Appl. Anal. 85(6-7), 781-791 (2006)

[21] King, J.P.: Positive linear operators which preserves $x^{2}$,. Acta Math. Hungar. 99, 203-208 (2003)

[22] Korovkin, P.P.: On convergence of linear positive operators in the spaces of continuous functions (Russian). Doklady Akad. Nauk. S.S.S.R. 90, 961-964 (1953)

[23] Korovkin, P.P.: Linear Operators and Approximation Theory. Hindustan, Delhi (1960)

[24] Schumaker, L.L.: Spline Functions: Basic Theory. Wiley, New York (1981)

[25] Wachnicki, E., Krech, G.: Approximation of functions by nonlinear integral operators depending on two parameters. Publ. Math. Debrecen 92(3-4), 481494 (2018)

[26] Yilmaz, B., Uysal, G., Aral, A.: Reconstruction of two approximation processes in order to reproduce $e^{a x}$ and $e^{2 a x}, a>0$. J. Math. Inequal. (2021) (to appear)

[27] Yuksel, I., Ispir, N.: Weighted approximation by a certain family of summation integral-type operators. Comput. Math. Appl. 52(10-11), 1463-1470 (2006)

Carlo Bardaro and Ilaria Mantellini

Department of Mathematics and Computer Sciences

University of Perugia

Via Vanvitelli, 1

06123 Perugia

Italy

e-mail: ilaria.mantellini@unipg.it

Carlo Bardaro

e-mail: carlo.bardaro@unipg.it

Gumrah Uysal

Division of Technology of Information Security, Department of Computer Technol-

ogy

Karabuk University

Karabuk

Turkey

e-mail: fgumrahuysal@gmail.com

Basar Yilmaz

Department of Mathematics, Faculty of Science and Arts

Kirikkale University

Yahsihan Kirikkale

Turkey

e-mail: basaryilmaz77@yahoo.com

Received: September 27, 2020.

Revised: May 12, 2021.

Accepted: June 2, 2021. 